\title{
An Adaptive Model for Optimizing Performance of an Incremental Web Crawler
}

\author{
Jenny Edwards* \\ Faculty of Information \\ Technology \\ University of Technology, \\ Sydney \\ PO Box 123 \\ Broadway NSW 2007 \\ Australia \\ jennycuit.uts.edurau
}

\author{
Kevin McCurley \\ IBM Research Division \\ Almaden Research Center, \\ $\mathrm{K} 53 / 802$ \\ 650 Harry Road \\ San Jose, CA 95120-6099 \\ USA \\ merurley'cialmaden.ibm.com
}

\author{
John Tomlin \\ IBM Research Division \\ Almaden Research Center, \\ $\mathrm{K} 53 / 802$ \\ 650 Harry Road \\ San Jose, CA 95120-6099 \\ USA \\ tomlin@almaden.ibm.com
}

\begin{abstract}
This paper out lines the design of a web crawler implemented for IB.1 Almaden s Wobfountain project and describes an optimization morlel for controlling the crawl strategy. This crawler is scalable and incremental. The morlel makes mo assimptioms about the statistical bohasione of web page changes. but rather uses an arlaptive approach to maintain data on actual change rates which are in turn used as inputs for the optimization. Computational results with simulated but realistic data show that there is no 'magic bullet' - different. but equally plansiblr. objectires lead to conflicting 'optimal strategies. However. we find that there are compromise objectives which lead to good strategies that are robust against a number of criteria.
\end{abstract}

\section{Categories and Subject Descriptors}

H.3.4 [Systems and Software]: Performance Evaluation (efficiency and effectiveness): $\mathrm{H}+3$ [Communications Applications]: Information Browsers: G1.6 [Optimization]: Nonlinear Programming

\section{General Terms}

Algorithms. Experimentation. Performance

\section{Keywords}

Crawler, incremental crawler. scalability optimization

*This work was crmpleted while the author was on leave at IB.I Almarlon Research Conter.

\section{INTRODUCTION}

Web crawlers are an essential component of all search engines, and are increasingly becoming important in clata mining and other indexing applications, but they must. function somewhere betwen the cushion of Monre's Law and the hard place of the exponential growth of the wob. While some have questioned whether such exponential growth is currently being maintained [1], the trend towards automated production of web pages from databases makes it likely that such growth will continue, or even arcelerate, in the immediate future. Given that the bandwidth for conducting crawls is neither infinite nor free it is becoming essential to crawl the web in a not only scalable, but efficient way if some reasonable measure of quality or freshness is to be maintained.

The WebFountain crawler to be outlined in this paper is designed to crawl the entire web repeatedly, keeping a local ropy of up to 1 MB of the text of each page, plus metadata, in a repository, which is to be used for indexing, mining, etc. Furthermore, this crawler is incremental, that is, the repository copy of each page is updated as soon as the actual web page is crawled. However, even using this technique, the repository must always be out of date to some (we hope minimal) extent.

Our crawler, to be described in greater detail below, has as an essential feature a set of queues of URLs to be crawled, and several parameters which determine how URLs are to be selected from these queues. The values of these parameters must be determined in a way that leads to efficient crawling, and we present a model which computes these parameters in such a way as to optinize some objective related to freshness. This model assumes that the web is crawled for some variable, but pre-specified number of discretized time periods which together constitute a cycle of the crawler.

Several definitions of freshness, which is non-trivial to measure, have been proposed by various authors, but in this paper we take the view that a page in the repository is either up-to-(late, or obsolcte, that is, it no longer matches the page on the real wob. Clearly it is desirable to minimize the number of such pages, but we shall sec that such an objective can be formulated in a number of ways. Pages become olsolete when they change on the real web between crawls, but for simplicity we shall also consider the special case of new pages, of which we have no copy in the repository, but 
which we become aware, either through new links, or exgenous information, and define them as also being obsolete.

A particular feature of the model we describe is that it

makes no a priori assumptions about the way in which pages change, simply that we can measure when changes occar, and record the frequency with the pages' metadata. In this sense the model is adaptive in that the more cycles the crawler is in operation, the more reliable and refined is the tata which we have available to drive it. For our purposes a page change is required to be non-trivial, as determined by a shingle (see [4]). Furthermore, while growth in the web wanges the data in our model, it has no effect on the size of the model nor the solution time.

No previous work that we know of makes use of precisely this set of assumptions, but much of it has some bearing on our model. After a review of this work, we present a more detailed description of the crawler, followed by a mathematical description of the optimization model for the model parameters. We then describe a series of computational experiments designed to test use of the model and some of its variants on simulated but realistic data

\section{PREVIOUS WORK}

There have been several studies of web crawling in its relatively short history, but most of them have had a focus rather different from ours. Some have concentrated on aspects relating to caching, e.g., [13] and [9]. Others have been principally interested in the most efficient and effective way to update a fixed size database extracted from the web, often for some specific function, such as data mining, see eg the work of Cho et al. $[5,6,7]$. These studies were performed over time periods ranging from a few days to seven months. However, for differing practical reasons, these crawlers were restricted to subsets of web pages.

Several authors, e.g., Coffman et al. [8], approach crawling from a theoretical point of view, comparing it to the polling systems of queueing theory, i.e., multiple queue-single server systems. However, the problem equivalent to the obsolescence time of a page is unexplored in the queueing litature.

A common assumption has been that page changes are a Poisson or memoryless process, with parameter $\lambda$ as the rate of change for the pages. Brewington and Cybenko [1] and Cho and Garcia-Molina [6] confirm this within the limits of their data gathering. This is somewhat undermined by another study, based on an extensive subset of the web by Brewington and Cybenko [2] showing that most web pages are modified during US working hours, ie $5 \mathrm{am}$ to $5 \mathrm{pm}$ (Silicon Valley Standard Time), Monday to Friday. Nevertheless, the widely accepted Poisson model forms the basis for a series of studies on crawler strategies. These lead to a variety of analytical models designed to minimize the age or maximize the freshness of a collection by investigating

- how often a page should be crawled

- in what order pages should be crawled

- should a crawling strategy be based on the importance of pages or their rates of change?

The definitions or metrics for freshness, age and imporance are not completely consistent, but in general the fresh- ness of a page refers to the difference between the time of crawling a page and the current time, while the age of a page is the difference between the time when the page last changed and the current time. Widely differing metrics have been offered for the importance of a page. but as the total mumber of possible metrics is large and the crawler in this study does not currently use any of them, no formal definitions will be given here.

While differing in detail, the experimental results in the referenced papers agree on general trends, and in particular that:

- the average size of individual pages is growing

- the proportion of visual and other nontextual material is growing in comparison to text

- the number of pages has been growing exponentially ([1] gives two different estimates of 318 and 390 days for the web to double in size but also says that the growth rate is slowing. However, see our comments at the beginning of Section 1.)

- different domains have very different page change rates

- the average age and lifetimes of pages are still quite low (cf Tables 1 and 2).

While all these trends are important for caching, the last three are more relevant to the study of whole web crawling to be discussed here.

\subsection{Crawling Models}

In a series of papers, Cho et al. [5, 6, 7] address a number of issues relating to the design of effective crawlers. In [i] they examine different crawling strategies using the Stanford University web pages as a particular subset of the web and examine several scenarios with different physical limitations on the crawling. Their approach is to visit more important pages first and they describe a number of possible metrics for determining this as well as the order in which the chosen pages will be visited. They show that it is possible to build crawlers which can obtain a significant portion of important pages quickly using a range of metrics. Their model appears to be most useful when trying to crawl large portions of the web with limited resources or if pages need to be revisited often to detect changes.

Cho and Garcia-Molina [6] derivet a series of mathematical models to determine the optimum strategy in a number of crawling scenarios, where the repusitory's extracted copy of the web is of fixed size. They examine models where all pages are deemed to change at the same average or uriform rate and where they change at different or non-uniform rates. For a real life crawler, the latter is more likely to be relevant. For each of these two cases. they examine a variety of synchronization or crawling policies. How often the repository web copy is updated depends on the crawling capacity or bandwidth available for the required number of pages. Within that limitation is the question of how often each individual page should be crawled to meet a particular objective such as maximizing freshness. Cho and GarciaMolina examine a uniform allocation policy. in which each 
page is crawled at the same rate, and a non-uniform or proportional policy where each page is crawled with a frequency that is propurtional to the frequency with which it is changed on the web. ie pages which are updated frequently are crawled more oft en than those which change only occasionally. Finally, they examine the order in which the pages should be crawled. They develop models of:

- fired order where all pages are crawled repeatedly in the same order each cycle

- randonn order where all pages are crawled in each cycle but in a random order. eg by always starting with the ront LRL for a site and crawling all pages linked to it

- purely random where pages are crawled on demand, which may mean some pages are crawled frequently and others never.

Their models show that when pages change at a uniform rate. maximum freshness or minimum age is obtained by crawling pages at a uniform rate and in fixed order. As we have noted, most studies make the assumption that pages change at a variable rate which may be approximated by a Poisson distribution, but in the second stage of their study, Cho and Garcia-Molina assume the broader gamma distribution for this change rate. Moreover, they prove that their particular model is valid for any distribution, and conclude that when pages change at varying rates. it is alwars better to crawl these pages at a uniform rate, ie ignoring the rate of change, than at a rate which is proportional to the rate of change. However, to maximize freshness they find a closed form solution to their model which provides an optimal crawling rate which is better than the uniform rate. These results were all derived for a batch or periodic crawler, is where a fixed number of pages is crawled in a given time period. These pages are used to update a fixed size repository either by replacing existing repository pages with newer versions or by replacing less important pages with those deemed to be of greater importance.

Coffman et al. [8] built a theoretical model to minimize the fraction of time pages spend out of date. Also assuming Poisson page change processes and a general distribution for page access time. they similarly show that optimal results can be obtained by crawling pages as uniformly as possible.

In [5], Cho and Garcia-Molina devise an architecture for an incremental crawler, and examine the use of an incremental versus a batch crawler under various conditions. particularly those where the entire web is not crawled. Crawling a subset ( 720.000 pages from 270 sites) of the web daily. they determined statistics on the rate of change of pages from different domains. They found. for example, that for the sites in their surver, $40 \%$ of pages in the com domain change daily in contrast to edu and gov domains where more than $50 \%$ of pages did not change in the four months of the study. They show that the rates of change of pages they crawled ran be approximated br a Poisson distribution, with the proviso that the figures for pages which change more often than daily or less often than four monthly are inaccurate. Using different collection procedures, Wills and Mikhailov [13] derive similar conclusions.

A disadvantage of all these models is that they deal only with a fixed size repository of a limited subset of the web. In contrast, our model is flexible, adaptive, based upon the whole web and caters gracefully for its growth.
Table 1: Cumulative Probability Distribution of Page Age in Days

\begin{tabular}{|c|c|}
\hline cumulative probability & page age (days) \\
\hline \hline 0.03 & $10^{0}$ \\
\hline 0.14 & $10^{1}$ \\
\hline 0.48 & $10^{2}$ \\
\hline 0.98 & $10^{3}$ \\
\hline
\end{tabular}

Table 2: Cumulative Probability Distribution of Mean Lifetime in Days

\begin{tabular}{|c|c|}
\hline cumulative probability & mean lifetime (days) \\
\hline \hline 0.0 & $10^{0}$ \\
\hline 0.12 & $10^{1}$ \\
\hline 0.40 & $10^{2}$ \\
\hline 1.00 & $.6 * 10^{3}$ \\
\hline
\end{tabular}

\subsection{Page Statistics Derived from Crawling}

Statistics on page ages, lifetimes, rates of change, etc are important for our model. Subject to the assumptions of a constant size repository copy of the web, which is updated with periodic uniform reindexing, Brewington and Cybenko [1] showed that in order to be sure that a randomly chosen page is at least $95 \%$ fresh or current up to a day ago, the web (of $800 \mathrm{M}$ ) pages needs a reindexing period of 8.5 days, and a reindexing period of 18 days is needed to be $95 \%$ sure that the repository copy of a random page was current up to 1 week ago. In another study [2], the same authors estimate the cumulative probability function for the page age in days on a $\log$ scale as shown in Table 1.

As with similar studies, this does not accurately account for pages which change very frequently or those which change very slowly. Allowing for these biases, the authors also estimate the cumulative probability of mean lifetime in days shown in Table 2.

Brewington and Cybenko then use their data to examine various reindexing strategies based on a single revisit period for all pages, and refer to the need for mathematical opti. mization to determine the optimal reindexing strategy when the reindexing period varies per page. Such a model is the main focus of this paper.

\section{THE WEBFOUNTAIN CRAWLER}

The model in this paper is designed to address the efficiency of a crawler recently built at IBM as part of the WebFountain data mining architecture. The features of this crawler that distinguish it from most previous crawlers are that it is fully distributed and incremental. By distributed, we mean that the responsibility for scheduling, fetching, parsing and storing is distributed anong a homogeneous cluster of machines. URLs are grouped by site, and a site is assigned to a machine in the cluster (a few very large sites such as geocities may actually be split among several machines). There is no global scheduler, nor are there any 
global queues to be maintained. Moreover, there is no machine with arcess to a global list of URLs.

An incremental crawler (as opposed to a batch crawler) is one that runs as an ongoing process, and the crawl is never regarded as complete. The underlying philosoplyy is that the local collection of documents will always grow, always be dynamic, and should be constructed with the goal of keeping the repository as fresh and complete as possible. Instead of devoting all of its effort to crawling newly discovered pages, a percentage of its time is devoted to recrawling pages that were crawled in the past, in order to minimize the number of obsolete pages. Note that our use of the term 'incremental' differs from that of Cho et al. $[5,6,7]$. Their definition assumes that the document collection is of static size, and a ranking function is used to replace documents in the collection with more important documents. We regard the issue of incrementality to be independent of the size of the collection, and we allow for growth of the crawler, in order to meet the demands of an ever-expanding web.

The WebFountain crawler is written in $\mathrm{C}++$, is fully distributed, and uses MPI (Message Passing Interface) for communication between the different components. The three major components are the Ants, which are the machines assigned to crawl sites, duplicate detectors, which are responsible for detecting duplicates or near-duplicates, and a single machine called a Controller. The Controller is the control point for the machine cluster, and keeps a dynamic list of site assignments on the Ants. It is also responsible for routing messages for discovered URLs, and manages the overall crawl rate, monitoring of disk space, load balancing, etc.

Other crawlers have been written that distribute the load of crawling across a cluster, but they generally distribute the work in different ways. Due to the competitive nature of the Internet indexing and searching business, few details are available about the latest generation of crawlers. The first generation Google crawler [3] is apparently designed as a batch crawler, and is only partially distributed. It uses a single point of control for scheduling of URLs to be crawled. While this might appear convenient, it also provides a bottleneck for intelligent scheduling algorithms, since the scheduling of URLs to be crawled may potentially ved to touch a large amount of data (eg, robots.txt, politeness values, change rate data, DNS records, etc). Mercator [10] supports incremental crawling using priority values on URLs and interleaving crawling new and old URLs.

The scheduling mechanism of the WebFountain crawler resembles Mercator in that it is fully distributed, very flexible, and can even be changed on the fly. This enables efficient use of all crawling processors and their underlying network. The base software component for determining the ordering on URLs to be crawled consists of a composition of sequencers. Sequencers are software objects that implement a few simple methods to determine the current backlog, whether there are any URLs available to be crawled, and control of loading and flushing data structures to disk. Sequencers are then implemented according to different policies, including a simple FIFO queue or a priority queue. Other Sequencers are combiners, and implement a policy for joining sequencers. Examples include a round robin aggregator, or a priority aggregator that probabilistically selects from among several sequencers according to some weights. In addition, we use the Sequencer mechanism to implement the crawling politeness policy for a site. The ability to combine sequencers and

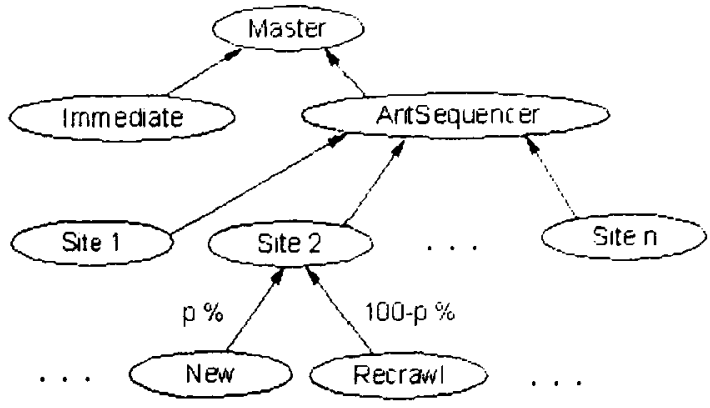

Figure 1: The Structure of Queues that Feed the URL Stream in the WebFountain Crawler

cascade them provides a very convenient means to build a flexible recrawl strategy.

The strategy that we decided on for inplementing the crawl strategy is illustrated in Figure 1. At the top level, there are two queues, one for immediate crawling that is intended to be used from a GUI, and one that aggregates all other URLs. Under that, each Ant is assigned a list of sites to be crawled, and maintains an active list of approximately 1000 sites that are currently being crawled. The selection of URLs to be crawled is taken from this active list in a round robin fashion. This avoids crawling any particular site too frequently - the so-called politeness criterion. In addition, each Ant is multithreaded to minimize lat ency effects. When a site is added to the active list, a sequencer is constructed that loads all data structures from disk, merges the list of newly discovered URLs with the list of previously crawled URLs for that site, and prepares two queues. One of these quenes contains URLs that have never been crawled. and one contains URLs that are scheduled for a recrawl. It is the way we manage our never-crawled and recrawl lists that it is to be determined by our optimisation model.

\section{MODEL DESCRIPTION}

We constructed this model for robustness under growth of the web and changes to its underlving nature. Hence we do not make any a priori assumptions about the distribution of page change rates. However, we do make an assumption that particular historical information on earle page is maintained in the metadat a for that page. Sperifically; and time a page is crawled, we record whether that page has changed since it was last crawled, and use this information to put the page into one of (at most 256 ) change-frequency buckets', recorded as one byte of the page's metarlata. C'learly. such dat a hecomes more reliable as the page ages. In prartice, page clange rates may range from as often as every 15 minutes to as infrequently as once a year or less (eg a government department contact page). The very rapidly changing pages (several times a clay) are almost all media sites (CNN, for example) and we feel that this relatively small and specialized set of sites should be handled separately. The remaining pages in the repository are grouped into $B$ buckets each containing $b_{i}$ pages which have similar rates of change. 


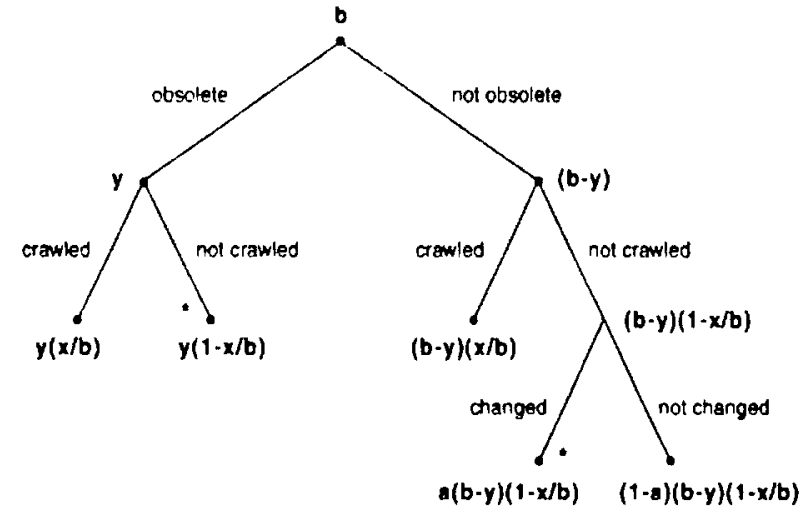

Figure 2: Identification of Obsolete Pages in each Bucket per Time Period

A (theoretically) complete crawl of the web is modeled as taking place in a crawler cycle. Both the number of time periods in such a cycle. $T$. and the (equal) length of each period may be varied as neerterl. Our fundamental rerpuirement is to estimate the number of obsolete pages in each frequency bucket at the end of each time period. The way in which this is calculated is best illustrated by following the tree of alternatives in Figure 2.

We consider a generic time period and a generic bucket (dropping the subscript) of pages in the repository, containing $b$ pages at the beginning of the time period. Let the number of such pages for which our repository copy is already obsolete at the beginning of the time period be $y$. leaving $(b-y)$ up-to-date. Now let $x$ be the number of pages in this bucket crawled during the time period, and assume that obsolete and up-to-rlate pages are crawled with equal probability. This gives the partition of pages in the bucket seen at the second level of branches in the tree. Finally, let $a$ be the (given) proportion of pages which change in the bucket during the time period. and assume that such a change is detected if the page is crawled in the same time period. The $b$ pages in the bucket are now partitioned among the leaves of the tree. and we easily see that the leaves marked * correspond to obsolete pages. Note that by adding the expressions attarhed to these two leaves we obtain an expression for the number of obsolete pages at the end of this time period. as a function of the data at the begimning of the period and of the decision variable $r$, which specifies how many pages in this bucket to crawl. This relationship) is fundamental to our morlel, and to the way in which the queue of 'old' LRLs is managed.

In addition to the 'old' pages we must deal with the 'new' pages either discovered or exogenously supplied during the rrawl. Let the number of these new pages crawled during a rime period be $=$ (these are then added to the appropriate buckets in the repository). The remaining new uncrawled pages are represented by $n$. These pages are also regarded as obsolete and will remain in that state until crawled.

We are now ready to give the definitions of the variables and the formal model.

$$
\begin{aligned}
& T=\text { number of time periods in model } \\
& B=\text { number of buckets } \\
& \text { where bucket refers to pages which change } \\
& \text { at approximately the same rate }
\end{aligned}
$$

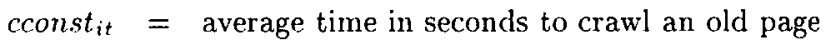

$$
\begin{aligned}
& \text { in bucket } i \text { in time period } t \\
& \text { dconst }_{t}=\text { average time in seconds to crawl a new page } \\
& \text { in time period } t \\
& \text { Cconst }_{t}=\text { total number of seconds available for crawling } \\
& \text { in time period } t \\
& \text { oldut }_{i t}=\text { experimental proportional weight on crawling } \\
& \text { obsolete pages in bucket } i \text { in time period } t \\
& \text { newwt }_{t}=\text { experimental proportional weight on crawling } \\
& \text { new pages in time period } t \\
& \text { oldnwt }_{i t}=\text { probability that when an old page in bucket } i \\
& \text { is crawled in time period } t \text {, it finds a new page } \\
& \text { neu'nut }_{1}=\text { probability that when a new uncrawled page } \\
& \text { is crawled in time period } t \text {, it finds a new page } \\
& \text { nonst }=\text { minimum number of new pages brought to the } \\
& \text { attention of the crawler per time period } \\
& b_{i t}=\text { number of pages in bucket } i \\
& \text { at the end of time period } t \\
& p_{i}=\text { distribution of new pages to buckets } \\
& \text { where } p_{i} \text { is proportional to } b_{i} \text { and } \sum_{i=1}^{B} p_{i}=1 \\
& a_{i t}=\text { fraction of pages in bucket } i \text { which change } \\
& \text { in time period } t \\
& y_{i t}=\text { number of obsolete pages in bucket } i \\
& \text { at end of time period } t \\
& x_{i t}=\text { number of crawled pages in bucket } i \\
& \text { in time period } t \\
& n_{t}=\text { number of new uncrawled pages } \\
& \text { at end of time period } t \\
& z_{t}=\text { number of new pages crawled in time period } t \text {. }
\end{aligned}
$$

The objective of the time period model is to minimize the weighted sum of obsolete pages, ie:

minimize

$$
\sum_{t=1}^{T}\left(\sum_{i=1}^{B} \text { oldwt } t_{i t} y_{i t}+n e w w t_{t} n_{t}\right)
$$

subject to the following constraints:

The bandwidth available for crawling during each time period may not be exceeded.

$$
\text { C const }_{t} \geq \sum_{i=1}^{B} \text { cconst }_{i t} x_{i t}+\text { dconst }_{t} z_{t}
$$

The number of obsolete existing or old pages, $y_{i t}$ is updated 
as discussed above at every time period.

$$
y_{i t}=\left(1-\left(x_{i t} / b_{i, t-1}\right)\right)\left(\left(1-a_{i t}\right) y_{i, t-1}+a_{i t} b_{i, t-1}\right)
$$

The number of new pages, $n_{t}$ is updated every time period.

$$
n_{t}=n_{t-1}+\sum_{i=1}^{B} \text { oldnut }_{i t} x_{t}+\left(\text { newnut } t_{t-1}\right) z_{t}+n \operatorname{con}_{\mathrm{s}}
$$

The total number of pages in each bucket, $b_{i t}$ is updated every time period.

$$
b_{i t}=b_{i, t-1}+p_{i} z_{t}
$$

The number of existing pages, $x_{i t}$ crawled in any bucket in any time period must be fewer than the total number of pages to be crawled in the bucket, $b_{i, t-1}$.

$$
x_{i t} \leq b_{i, t-1}
$$

Similarly, in any time period, the number of new pages crawled, $z_{t}$ must be less than the number of new uncrawled 'ges, $n_{t-1}$.

$$
\begin{gathered}
z_{t} \leq n_{t-1} \\
0 \leq b_{i t}, x_{i t}, y_{i t}, n_{t}, z_{t}
\end{gathered}
$$

Each equation holds $\forall t=1, \ldots, T$ and, where relevant, $\forall i=1, \ldots, B$

The critical solution outputs are the ratios $x_{i t} / b_{i t}$ and the $z_{t}$ values, which tell us how many URLs in the 'old' and 'new' queues we should crawl in each time period, and hence the probabilities $p$ in Figure 1.

\section{SOLUTION}

Since the balance equation 2 for updating $y_{i t}$ is highly nonlinear, the model must be solved by a nonlinear programming (NLP) method capable of solving large scale problems. For our model runs, it was assumed that there are $500 \mathrm{M}$ unique pages on the web, with a growth rate which allows the web to double in size in approximately 400 days. With a $v^{2}$ '. 1 number of buckets, the basic model has approximately 11200 variables of which 10000 are nonlinear and 11200 constraints of which about 3300 are nonlinear. Even after the use of various presolve techniques to reduce the size of the problem, the solution proved to be non-trivial.

We used the NEOS [12] public server system to run experiments with several different NLP solvers on the models and found that the standard NLP package, MINOS [11] gave the best and most reliable results. Solution times for all variations of the model were around ten minutes on MINOS on a timeshared machine.

Since the WebFountain crawler for which this model was designed is in its early stages of development, we have very little actual historical data for such parameters as the rate of change of various pages. We have, therefore, used simulated, but realistic data, based on the previous studies we have cited from the literature.

\subsection{Results}

Since our model runs use estimated data, many experiments were run for a range of values of the critical parameters. Reassuringly, the model is quite robust under most of
Table 3: Model Statistics

\begin{tabular}{|c|c|c|c|}
\hline & objective & $\begin{array}{c}\text { Total Pages } \\
\text { at end }\end{array}$ & $\begin{array}{c}\text { Total Obsolete } \\
\text { Pages at cind }\end{array}$ \\
\hline \hline Strat1 & $32.592 \times 10^{7}$ & $5.224 \times 10^{7}$ & $3.086 \times 10^{7}$ \\
\hline Strat2 & $1.722 \times 10^{7}$ & $5.184 \times 10^{7}$ & $1.609 \times 10^{7}$ \\
\hline Strat3 & $2.138 \times 10^{7}$ & $5.224 \times 10^{7}$ & $1.702 \times 10^{2}$ \\
\hline
\end{tabular}

these changes. However, it turns out to be quite sensitive to changes in the weights in the objective function, oldat at and newe $t_{t}$. Given the overall ain of minimizing the total number of obsolete pages, we describe the implentent at ion of three of the many possible variations of the objective function weights:

- Strategy 1 gives equal weights (summing to 1) to each time period in a cycle of the crawler

- Strategy 2 gives the last time period the total weight of 1 and the other time periods, zero weight, ie the model is minimizing the number of obsolete pages just on the last time period of a crawler cycle

- Strategy 3 gives the last time period a high weighting and the remaining time periods very low weightings, ie it is still trying to minimize the number of obsolete pages in the last time period but it is also taking into account the obsolete pages in all time periods.

\subsection{Experiment 1}

As shown in Table 3, Strategy 2 gives the minimum value of the objective function, ie the weighted total of obsolete pages, followed by Strategy 3. The objective value for Strategy 1 is considerably higher. Figure 3 illustrates the effects of implementing these strategies.

Strategy 2 recommends crawling each page just once during a cycle of the crawler. This uniform crawling is in line with the theoretical results of Cho and Garcia-Molina [6] and Coffman et al. [8]. Strategy 1 recommends crawling fast changing pages in many time periods of a cycle. For these pages, the crawl rate is usually higher exen than the prage change rate. However, it does not crawl at all, those pages which fall into the lowest $40 \%$ of page change rates. Strategy 3 is a compromise. It recommends crawling all pages at least once in a crawler cycle with the fastest changing $18 \%$ being crawled more than once per cycle.

Table 3 also shows that while the total number of web pages at the end of a crawler cycle is similar under each strategy, the total number of obsolete pages is not. Figure 1 examines the total number of obsolete pages in each time period of a cycle under each strategy:

If we disregard the actual objective function and look at the number of obsolete pages we see that in any given time period (except the last), Strategy 1 always has fewer obsolete pages than Strategy 3 and considerably fewer than Strategy 2. Because of the weights in the objective functions for the different strategies, the lower number of obsolete pages for Strategies 2 and 3 in the last time period is expected.

Mathematically, Strategy 2 is optimal. However, depending on the purpose(s) for which a crawler is designeel, it may not be acceptable to have an incremental crawler ignore the page change rate and crawl all pages at a uniform 


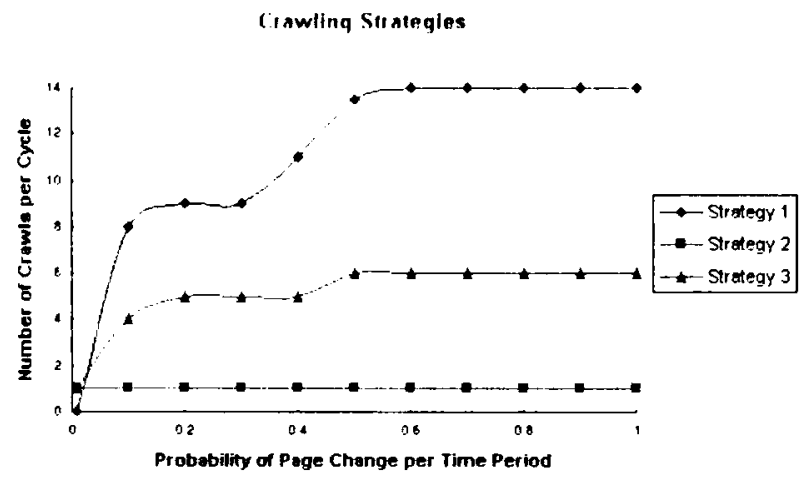

Figure 3: Recommended Number of Crawls per Crawler Cycle for Pages with Different Change Rates under Different Crawl Strategies

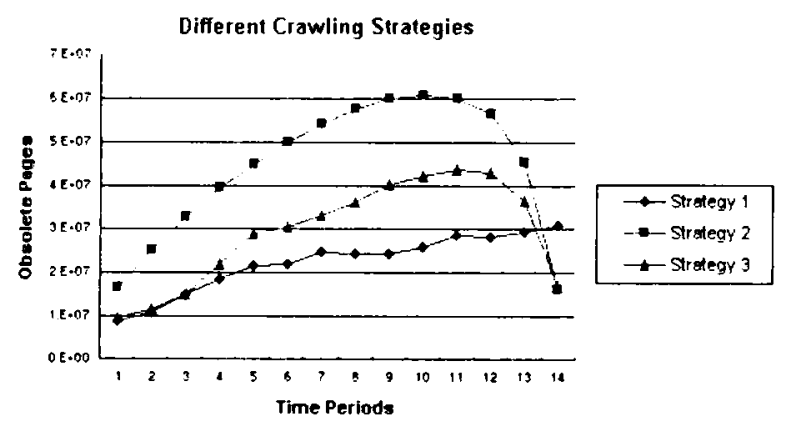

Figure 4: Total Number of Obsolete Pages each Time Period under Different Strategies
Table 4: Model Statistics for Versions 1 and 2 of Strategies 1 and 3

\begin{tabular}{|c|c|c|c|}
\hline & objective & $\begin{array}{c}\text { Total Pages } \\
\text { at end }\end{array}$ & $\begin{array}{c}\text { Total Obsolete } \\
\text { Pages at end }\end{array}$ \\
\hline \hline S1 v1 & $32.592 \times 10^{7}$ & $5.224 \times 10^{8}$ & $3.086 \times 10^{7}$ \\
\hline S1v2 & $37.744 \times 10^{7}$ & $5.214 \times 10^{8}$ & $3.534 \times 10^{7}$ \\
\hline S3v1 & $2.138 \times 10^{7}$ & $5.224 \times 10^{9}$ & $1.702 \times 10^{8}$ \\
\hline S3v2 & $2.500 \times 10^{7}$ & $5.193 \times 10^{9}$ & $1.972 \times 10^{8}$ \\
\hline
\end{tabular}

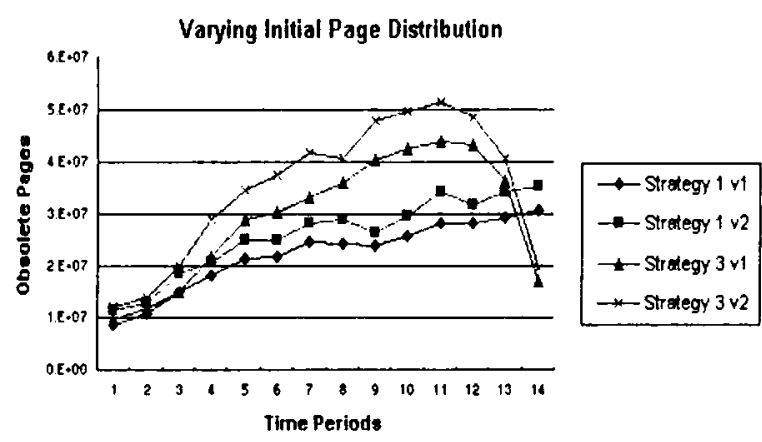

Figure 5: Effects on the Number of Obsolete Pages per Time Period of Varying the Page Change Rates Distribution

rate, ie just once each crawler cycle. In terms of minimizing the number of obsolete pages each time period, Strategy 1 is clearly superior. However, it does not crawl $40 \%$ of the pages during a cycle. This may also be unacceptable for many of the possible uses of the crawler.

Strategy 3 again provides a reasonable compromise. It crawls all pages within a cycle and still has a somewhat lower mathematical ininimum objective value than Strategy 1 .

\subsection{Experiment 2}

Many versions of the model were run changing the values of different parameters. In all cases the results were as expected. Experiment 2 is a typical example. In the initial or Version 1 of the model, the $500 \mathrm{M}$ repository pages were assumed to be distributed equally to each bucket, ie it was assumed that there are the same number of pages corresponding to each of the different page change rates. Each of the 255 buckets received $2 \mathrm{M}$ pages. In Version 2, the buckets representing the $25 \%$ of the fastest changing page rates and the $25 \%$ of the slowest changing pages all received $3 \mathrm{M}$ pages initially. The buckets representing the middle $50 \%$ of page change rates, each received $1 \mathrm{M}$ pages initially. Table 4 shows the effect on the total number of obsolete pages of this change.

Figure 5 shows that this change made little difference to the distribution of obsolete pages for each time period. For both Strategy 1 and Strategy 3 , there are a larger number of obsolete pages in Version 2. This is expected as the models in Version 2 started with a higher number of obsolete pages and given the finite capacity of the crawler, this number will grow during a 14 time period cycle of the crawler unless forced to reduce as in the last few time periods of Strategy 3. 


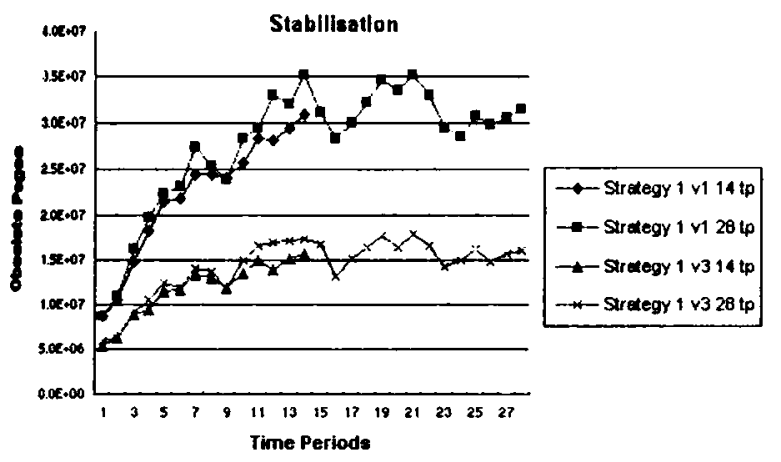

Figure 6: Trend towards Stabilisation in the Number of Obsolete Pages over Time

Experiment 2 does show the robustness of the model to changes in the initial parameters.

\subsection{Experiment 3}

The object of Experiment 3 was to determine if the number of obsolete pages continued to grow with time or if this number reached stabilisation. There were four runs, all of the model with an objective corresponding to Strategy 1. Figure 6 illustrates the results. Strategy 1 (Version 1) was run for 14 time periods and for 28 time periods as was Version 3. In Version 3, it was assumed that the page change rates were half as frequent as in Version 1. The distribution of obsolete pages over time periods between and within each version shows the expected similarities. As can be seen in any given time period, the number of obsolete pages for Version 3 is approximately half that of Version 1. More importantly, it can be seen that for both versions, the number of obsolete pages is tending to stabilise. It was not possible to run the crawler model for a longer period and obtain a useful mathematical solution, nor would the crawler be run for this long in practice without an update of the parameters and reoptimization.

The objectives we used, based on the different weighted ..ms of obsolete pages, correspond to maximising the freshness of the collection under different crawler aims, eg all the web must be crawled each cycle or a certain percentage of pages in the repository should be guaranteed to be no more than say, a week old.

Taking all the experiments into consideration, the results are consistent with an implementation philosophy of using Strategy 2 in early cycles of the crawler, to drive down the number of obsolete pages in the repository quickly. It would then be beneficial to switch to Strategy 1 or 3 to maintain a stable number.

\section{CONCLUSIONS}

The computational results we have obtained (albeit with simulated data) suggest that an efficient crawling strategy can be implemented for the WebFountain (or any) incremental crawler without making any theoretical assumptions about the rate of change of pages, but by using information gleaned from actual cycles of the crawler which adaptively build up more extensive and reliable data. Thus the model we have described is adaptive at two levels: within a crawler cycle it coordinates the management of the URL queues over the cycle's component time periods, and between cycles the data necessary for the optimization is updated for the next cycle - particularly the change rates, and new page creation rates. We look forward to full scale implementation of the model when the WebFountain crawler begins regular operation.

\section{ACKNOWLEDGEMENTS}

The authors would like to thank members of IBM's WebFountain team, in particular, Sridhar Rajagopalan and Andrew Tomkins. They would also like to thank Michael Saunders of Stanford University and the NEOS team for help with the solution of the nonlinear programming problems and Paula Wirth for technical assistance.

\section{REFERENCES}

[1] B. Brewington and G. Cybenko. How dynamic is the web? In Proceedings of the 9th World Wide Web Conference (WWWg), 2000.

[2] B. Brewington and G. Cybenko. Keeping up with the changing web. Computer, pages 52-58, May 2000.

[3] S. Brin and L. Page. The anatomy of a large-scale hypertextual web search engine. In Proceedings of the 7th World Wide Web Conference (W'WW 7 ), 1998.

[4] A. Broder, S. Glassman, M. Manasse, and G. Zweig. Syntactic clustering of the web. In Proceedings of 6 th International World Wide Web Conference (WWW6), 1997.

[5] J. Cho and H. Garcia-Molina. The evolution of the web and implications for an incremental crawler. In Proceedings of 26th International Conference on Very Large Databases (VLDB), 2000.

[6] J. Cho and H. Garcia-Molina. Synchronizing a database to improve freshness. In Proceedings of 2000 ACM International Conference on Management of Data (SIGMOD), 2000.

[7] J. Cho, H. Garcia-Molina, and L. Page. Efficient crawling through URL ordering. In Proceedings of the 7th World Wide Web Conference (W'WW'), 1998.

[8] E. Coffman, Z. Liu, and R. Weber. Optimal robot scheduling for web search engines, Rapport de recherche no 3317 . Technical report, INRIA Sophia Antipolis, 1997.

[9] F. Douglis, A. Feldmann, and B. Krishnamurthy. Rate of change and other metrics: a live study of the world wide web. In Proceedings of USENIX Symposium on Internetworking Technologies and Systems, 1997.

[10] A. Heydon and M. Najork. Mercator: A scalable, extensible web crawler. World Wide Web, 2(4):219-229, 1999.

[11] MINOS. (http://www.sbsi-sol-optimize.com/minos.htm).

[12] NEOS Server for Optimization. (http://www-neos.mcs.anl.gov).

[13] C. Wills and M. Mikhailov. Towards a better understanding of web resources and server responses for improved caching. In Proceedings of the 8th World Wide Web Conference (WWW8), 1999. 